\title{
Weighted Tensor Products of Joyal Species, Graphs, and Charades
}

\author{
Ross STREET
}

Centre of Australian Category Theory, Macquarie University, Australia

E-mail: ross.street@mq.edu.au

URL: http://maths.mq.edu.au/ street/

Received August 18, 2015, in final form January 14, 2016; Published online January 17, 2016

http://dx.doi.org/10.3842/SIGMA.2016.005

\begin{abstract}
Motivated by the weighted Hurwitz product on sequences in an algebra, we produce a family of monoidal structures on the category of Joyal species. We suggest a family of tensor products for charades. We begin by seeing weighted derivational algebras and weighted Rota-Baxter algebras as special monoids and special semigroups, respectively, for the same monoidal structure on the category of graphs in a monoidal additive category. Weighted derivations are lifted to the categorical level.
\end{abstract}

Key words: weighted derivation; Hurwitz series; monoidal category; Joyal species; convolution; Rota-Baxter operator

2010 Mathematics Subject Classification: 18D10; 05A15; 18A32; 18D05; 20H30; 16T30

\section{Introduction}

The weighted Hurwitz product arises naturally in the study of weighted derivations and weighted Rota-Baxter operators; see $[12,23]$ and their references. We review these concepts from a categorical perspective in Section 2.

In Section 3 we present a monoidal structure on the category Gph $\mathscr{V}$ of graphs in a monoidal additive category $\mathscr{V}$. We show how these derivations and operators on a monoid $A$ in $\mathscr{V}$ can be viewed as monoid and semigroup structures on particular graphs constructed from $A$.

The main theme of the paper is to lift concepts defined on algebras (or monoids) to concepts defined on monoidal categories ${ }^{1}$. The $\lambda$-weighted Hurwitz product is traditionally defined on the abelian group $A^{\mathbb{N}}$ of sequences in an algebra $A$. This lifts in an obvious way, for a set $\Lambda$, to a definition of $\Lambda$-weighted product on the category $\mathscr{V}^{\mathbb{N}}$ of sequences of objects in a reasonable monoidal $\mathscr{V}$. Rather than $\mathscr{V}^{\mathbb{N}}$, our interest here is in the category $[\mathfrak{S}, \mathscr{V}]$ of $\mathscr{V}$-valued Joyal species [13] and a weighted version of the convolution product, called Cauchy product in [2]. This is fully discussed in Sections 4-8 and 10, and motivates a definition of weighted categorical derivation in Section 9. The weighted tensors define an interesting family of tensor products for linear representations of the symmetric groups which, by choosing specific weights, include the Cauchy product and the Heisenberg product [20].

Finally, Sections 11 and 12 suggest and explore a weighted tensor product for species on finite vector spaces (or charades [18, 22]). This generalizes, in particular, the essentially classical tensor product of representations of the general linear groups over a finite field, proved braided in [17].

Further insights into the subject of this paper appear in [11].

\footnotetext{
${ }^{1}$ Some authors call this process "categorification".
} 


\section{Review of weighted derivations and Rota-Baxter operators}

The inspiration for the family of tensor products on species came from the $\lambda$-weighted product of Hurwitz series as discussed in $[12,23]$ and their references. They begin by defining a derivation of weight $\lambda$ on an algebra $A$ over a commutative ring $k$, with given $\lambda \in k$, to be a $k$-module morphism $d: A \rightarrow A$ satisfying $d(1)=0$ and

$$
d(a b)=d(a) b+a d(b)+\lambda d(a) d(b) .
$$

They note the generalized Leibnitz rule

$$
d^{n}(a b)=\sum_{k=0}^{n} \sum_{j=0}^{n-k}\left(\begin{array}{l}
n \\
k
\end{array}\right)\left(\begin{array}{c}
n-k \\
j
\end{array}\right) \lambda^{k} d^{n-j}(a) d^{k+j}(b) .
$$

However, I prefer to write this in the form

$$
d^{n}(a b)=\sum_{n=r+s+t}\left(\begin{array}{c}
n \\
r, s, t
\end{array}\right) \lambda^{t} d^{r+t}(a) d^{s+t}(b)
$$

to emphasise the relationship to the trinomial expansion rule for $(x+y+\lambda x y)^{n}$. Here

$$
\left(\begin{array}{c}
n \\
r, s, t
\end{array}\right)=\frac{n !}{r ! s ! t !} \text {. }
$$

The $\lambda$-Hurwitz product on $A^{\mathbb{N}}$ can be defined by the clearly related equation

$$
\left(f \cdot \cdot^{\lambda} g\right)(n)=\sum_{n=r+s+t}\left(\begin{array}{c}
n \\
r, s, t
\end{array}\right) \lambda^{t} f(r+t) g(s+t) .
$$

Example 2.1. For $\lambda=0, k=\mathbb{R}$ and $A$ the algebra of smooth functions $f: \mathbb{R} \rightarrow \mathbb{R}$ under pointwise addition and multiplication, the differentiation function $d: A \rightarrow A$ is a 0 -weighted derivation by the classical Leibnitz rule.

Example 2.2. For $\lambda$ invertible, $k=\mathbb{R}$ and $A$ the algebra of functions $f: \mathbb{R} \rightarrow \mathbb{R}$ under pointwise addition and multiplication, the function $d: A \rightarrow A$ defined by

$$
d(f)(x)=\frac{f(x+\lambda)-f(x)}{\lambda}
$$

is a $\lambda$-weighted derivation.

Example 2.3. Define $d: A^{\mathbb{N}} \rightarrow A^{\mathbb{N}}$ by $d(s)(n)=s(n+1)-s(n)$. This $d$ is a 1-weighted derivation when $A^{\mathbb{N}}$ is equipped with the pointwise addition and multiplication.

Example 2.4. Define $d: A^{\mathbb{N}} \rightarrow A^{\mathbb{N}}$ by $d(f)(n)=f(n+1)$. This $d$ is a $\lambda$-weighted derivation when $A^{\mathbb{N}}$ is equipped with the $\lambda$-Hurwitz product for any $\lambda$. Notice that we have an algebra morphism $d^{*}: A^{\mathbb{N}} \rightarrow\left(A^{\mathbb{N}}\right)^{\mathbb{N}}$ defined by $d^{*}(f)(m)(n)=f(m+n)$. This may motivate the next definition.

Define $d^{*}: A \rightarrow A^{\mathbb{N}}$ by $d^{*}(a)(n)=d^{n}(a)$. We see that the Leibnitz rule (2.1) amounts to

Proposition 2.5. $d^{*}: A \rightarrow A^{\mathbb{N}}$ is an algebra morphism for all $\lambda$-weighted derivations $d$ on $A$, where $A^{\mathbb{N}}$ has the $\lambda$-Hurwitz product. 
In fact, $A \mapsto A^{\mathbb{N}}$ is a comonad

$$
G=\left((-)^{\mathbb{N}}, \varepsilon, \delta\right)
$$

on the category $\mathrm{Alg}_{k}$ of $k$-algebras whose Eilenberg-Moore-coalgebras are $k$-algebras $A$ equipped with a $\lambda$-derivation, so-called $\lambda$-derivation algebras; write $\mathrm{DA}_{\lambda}$ for the category of these. The morphism $d^{*}: A \rightarrow A^{\mathbb{N}}$ is the coaction of the comonad.

Where there is differentiation, there should also be integration. A Rota-Baxter operator of weight $\lambda$ on a $k$-algebra $A$ is a $k$-linear morphism $P: A \rightarrow A$ satisfying

$$
P(a) P(b)=P(P(a) b+P(a) b+\lambda a b) .
$$

The pair $(A, P)$ is called a $\lambda$-weighted Rota-Baxter algebra. Write $\mathrm{RBA}_{\lambda}$ for the category of these.

Example 2.6. For $\lambda=0, k=\mathbb{R}$ and $A$ the algebra of continuous functions $f: \mathbb{R} \rightarrow \mathbb{R}$ under pointwise addition and multiplication, the integration function $P: A \rightarrow A$, defined by $P(f)(x)=$ $\int_{0}^{x} f(t) \mathrm{d} t$, is a 0 -weighted Rota-Baxter operator by the classical integration-by-parts rule.

Example 2.7. For $\lambda=1$ and any $k$-algebra $A$, define $P: A^{\mathbb{N}} \rightarrow A^{\mathbb{N}}$ to take a sequence $u$ in $A$ to its sequence $P(u)$ of partial sums

$$
P(u)(n)=\sum_{i=0}^{n-1} u(i) .
$$

Then $P$ is a 1 -weighted Rota-Baxter operator on $A^{\mathbb{N}}$ with pointwise addition and multiplication. See $[4,21]$. For $d$ the consecutive difference operator as defined in Example 2.3, notice that $d \circ P=1_{A^{\mathbb{N}}}$.

Example 2.8. If $Q$ is a 1-weighted Rota-Baxter operator on $A$ then $P(a)=\lambda Q(a)$ defines a $\lambda$-weighted Rota-Baxter operator $P$ on $A$.

A $\lambda$-weighted derivation $R B$-algebra is a $k$-algebra $A$ equipped with a $\lambda$-weighted derivation $d$ and a $\lambda$-weighted Rota-Baxter operator $P$ such that $d \circ P=1_{A}$. Write $\mathrm{DRB}_{\lambda}$ for the category of these.

Proposition 2.9 (see [12]). Let $P$ be a $R B$-operator of weight $\lambda$ on $A$. Then $A^{\mathbb{N}}$ equipped with the $\lambda$-Hurwitz product, the derivation $d$ of Example 2.4, and $P$ defined by

$$
P(f)(n)= \begin{cases}P(f(0)) & \text { for } n=0, \\ f(n-1) & \text { for } n>0\end{cases}
$$

is a $\lambda$-weighted derivation RB-algebra. Moreover, the following square commutes

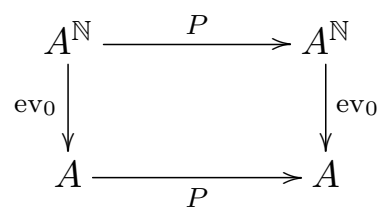

With a little more work following Proposition 2.9, we see that the comonad $G(2.3)$ lifts to $\mathrm{RBA}_{\lambda}$. In particular, with $\mathrm{V}$ denoting the forgetful functor, we have a comonad $\bar{G}$ and a commutative square

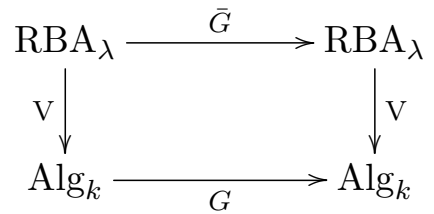


Write $\mathrm{RBA}_{\lambda-}$ for the category of $\lambda$-weighted Rota-Baxter algebras where we do not insist on the algebras having a unit.

Proposition 2.10. For each $(A, \cdot, P) \in \mathrm{RBA}_{\lambda_{-}}$, there is an associative binary operation $a \diamond b$ defined on $A$ by

$$
a \diamond b=P(a) \cdot b+a \cdot P(b)+\lambda a \cdot b .
$$

Moreover, $T(A, \cdot, P)=(A, \diamond, P)$ defines an endofunctor

$$
T: \mathrm{RBA}_{\lambda_{-}} \longrightarrow \mathrm{RBA}_{\lambda_{-}},
$$

which is (well-)copointed by a natural transformation $\gamma: T \Rightarrow 1_{\mathrm{RBA}_{\lambda_{-}}}$whose component at $(A, \cdot, P)$ is $P:(A, \diamond, P) \rightarrow(A, \cdot, P)$.

Remark 2.11. The day after my seminar talk of 4 February 2015, on the material of this section and Section 4, Stephen Lack made the following comments:

1. Consider the category $[\Sigma \mathbb{N}, \mathscr{V}]$ whose objects are pairs $(M, d)$ consisting of an object $M$ of a nice monoidal additive category $\mathscr{V}$ and an endomorphism $d: M \rightarrow M$. The forgetful functor

$$
\mathrm{U}:[\Sigma \mathbb{N}, \mathscr{V}] \longrightarrow \mathscr{V}
$$

taking $(M, d)$ to $M$, has a right adjoint taking $M$ to $\left(M^{\mathbb{N}}, d\right)$ where $d(f)(n)=f(n+1)$. There is a monoidal structure on $[\Sigma \mathbb{N}, \mathscr{V}]$ defined by

$$
(M, d) \otimes^{\lambda}(N, d)=(M \otimes N, d \otimes 1+1 \otimes d+\lambda d \otimes d) .
$$

The monoids in this monoidal category are precisely $\lambda$-derivation algebras. Moreover, $\mathrm{U}(2.4)$ and its right adjoint form a monoidal adjunction which therefore defines an adjunction between the categories of monoids. This adjunction generates the comonad $G(2.3)$ on the category Mon $\mathscr{V}$ of monoids in $\mathscr{V}$.

2. There is a bialgebra structure on the polynomial algebra $k[x]$ with comultiplication the algebra morphism $\delta: k[x] \rightarrow k[x, y] \cong k[x] \otimes k[x]$ defined by

$$
\delta(x)=x+y+\lambda x y .
$$

Then the convolution product on the left-hand side of the canonical isomorphism

$$
\operatorname{Mod}_{k}(k[x], A) \cong A^{\mathbb{N}}
$$

transports to the $\lambda$-Hurwitz product on $A^{\mathbb{N}}$.

3. It feels like there should be a multicategory/promonoidal/substitude structure on $[\Sigma \mathbb{N}, \mathscr{V}]$ for dealing with RB-algebras.

\section{$3 \quad$ Graphs in monoidal additive categories}

Let $\mathscr{V}$ be a monoidal additive category. We act as if the monoidal structure were strict.

Let Gph $\mathscr{V}$ be the category of directed graphs in $\mathscr{V}$. So an object has the form of a pair of parallel morphisms $s, t: E \longrightarrow A$ in $\mathscr{V}$; we use $s$ and $t$ for source and target morphisms in all 
graphs. A morphism $(f, \phi):(A, E) \longrightarrow(B, F)$ in Gph $\mathscr{V}$ consists of morphisms $f$ and $\phi$ making the following diagram commute

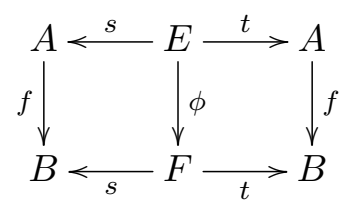

Write ver: Gph $\mathscr{V} \longrightarrow \mathscr{V}$ for the forgetful functor taking $(A, E)$ to $A$ and write edg: Gph $\mathscr{V} \longrightarrow \mathscr{V}$ for the forgetful functor taking $(A, E)$ to $E$.

We will use the notation $\langle n\rangle=\{1,2, \ldots, n\}$. For $R \subseteq\langle n\rangle$, write

$$
\chi_{R}:\langle n\rangle \longrightarrow\{s, t\}
$$

for the characteristic function of $R$ defined by

$$
\chi_{R}(i)= \begin{cases}s & \text { for } i \in R, \\ t & \text { for } i \notin R .\end{cases}
$$

Choose an endomorphism $\lambda: I \rightarrow I$ of the tensor unit $I$ in $\mathscr{V}$. For any $f: A \rightarrow B$ in $\mathscr{V}$, we define $(\lambda f: A \rightarrow B)=(\lambda \otimes f: I \otimes A \rightarrow I \otimes B)$.

Given a list $\left(A_{1}, E_{1}\right), \ldots,\left(A_{n}, E_{n}\right)$ of objects of Gph $\mathscr{V}$, we define an $n$-fold tensor product

$$
\otimes_{1 \leq i \leq n}^{\lambda}\left(A_{i}, E_{i}\right)=\left(\otimes_{1 \leq i \leq n} A_{i}, \otimes_{1 \leq i \leq n} E_{i}\right)
$$

where

$$
s=\sum_{\varnothing \neq R \subseteq\langle n\rangle} \lambda^{(\# R-1)} \chi_{R}(1) \otimes \cdots \otimes \chi_{R}(n) \quad \text { and } \quad t=t \otimes \cdots \otimes t .
$$

For $n=2$ this gives a binary tensor product

$$
(A, E) \otimes^{\lambda}(B, F)=(A \otimes B, E \otimes F)
$$

with

$$
s=\lambda s \otimes s+s \otimes t+t \otimes s \quad \text { and } \quad t=t \otimes t .
$$

The unit for this tensor is the graph $(I, I)$ with $s=0: I \rightarrow I$ and $t=1_{I}: I \rightarrow I$.

Proposition 3.1. A monoidal structure on $\mathrm{Gph} \mathscr{V}$ is defined by (3.1) for any given $\lambda \in \mathscr{V}(I, I)$. Both ver and edg: Gph $\mathscr{V} \longrightarrow \mathscr{V}$ are strict monoidal.

Proof. Easy calculations of the source morphisms for

$$
\left((A, E) \otimes^{\lambda}(B, F)\right) \otimes^{\lambda}(C, G) \quad \text { and } \quad(A, E) \otimes^{\lambda}\left((B, F) \otimes^{\lambda}(C, G)\right)
$$

show they agree with that of the triple tensor product. The target morphisms obviously agree. What this means is that the associativity constraints for $\mathscr{V}$ lift through ver and edg to Gph $\mathscr{V}$ and are therefore coherent.

Let $[\Sigma \mathbb{N}, \mathscr{V}]$ denote the category whose objects $(A, e: A \rightarrow A)$ consist of an object $A$ of $\mathscr{V}$ equipped with an endomorphism $e$. Let

$J:[\Sigma \mathbb{N}, \mathscr{V}] \longrightarrow \operatorname{Gph} \mathscr{V}$ 
be the functor defined by $J(A, e)=(A, A)$ with $s=e$ and $t=1_{A}$; and $J f=(f, f)$. Notice also that a morphism $(f, \phi):(B, F) \rightarrow J(A, e)$ in Gph $\mathscr{V}$ with codomain in the subcategory amounts to a commutative diagram

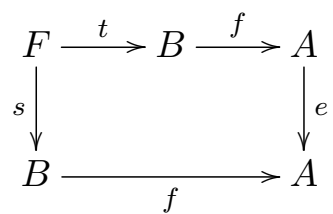

where $\phi$ is forced to be $f \circ t: F \rightarrow A$. Clearly $J$ is fully faithful and the monoidal structure of Proposition 3.1 restricts to a monoidal structure on $[\Sigma \mathbb{N}, \mathscr{V}]$ yielding (3.2) as a strict monoidal functor. Indeed, this is none other than the monoidal structure of Remark 2.11, item 1.

Definition 3.2. A $\lambda$-weighted derivational monoid in $\mathscr{V}$ is a monoid $(A, d)$ in $[\Sigma \mathbb{N}, \mathscr{V}]$ equipped with the monoidal structure obtained as the restriction through (3.2) of that of Proposition 3.1 on $\operatorname{Gph} \mathscr{V}$. An object $(A, d) \in[\Sigma \mathbb{N}, \mathscr{V}]$ with an associative binary operation will be called a $\lambda$-weighted derivational semigroup.

More explicitly, a $\lambda$-weighted derivational monoid is a monoid $A$ in $\mathscr{V}$ equipped with an endomorphism $d: A \rightarrow A$ satisfying the $\lambda$-weighted equation

$$
d \circ \mu=\mu \circ(\lambda d \otimes d+d \otimes 1+1 \otimes d),
$$

and the equation $d \circ \eta=0$ (where $\eta: I \rightarrow A$ is the unit of $A$ ).

There is an isomorphism of categories

$$
\text { op: } \operatorname{Gph} \mathscr{V} \longrightarrow \operatorname{Gph} \mathscr{V}
$$

taking $(A, E)$ to $(A, E)^{\mathrm{op}}$ for which $A$ and $E$ are unchanged but $s$ and $t$ have been interchanged.

Put

$$
J^{\mathrm{op}}=([\Sigma \mathbb{N}, \mathscr{V}] \stackrel{J}{\longrightarrow} \mathrm{Gph} \mathscr{V} \stackrel{\mathrm{op}}{\longrightarrow} \mathrm{Gph} \mathscr{V}) .
$$

Like $J$, this composite $J^{\text {op }}$ is fully faithful.

However, the image of $J^{\text {op }}$ is not closed under the monoidal structure of Proposition 3.1. All we obtain on $[\Sigma \mathbb{N}, \mathscr{V}]$ is a structure of multicategory (sometimes called a "coloured operad"). The sets of multimorphisms are defined by

$$
\mathrm{P}_{\lambda}\left(\left(A_{1}, p_{1}\right), \ldots,\left(A_{n}, p_{n}\right) ;(B, p)\right)=\mathrm{Gph} \mathscr{V}\left(\otimes_{1 \leq i \leq n}^{\lambda} J^{\mathrm{op}}\left(A_{i}, p_{i}\right), J^{\mathrm{op}}(B, p)\right) .
$$

To be more explicit, for $R \subseteq\langle n\rangle$ and $i \in\langle n\rangle$, put

$$
R(i)= \begin{cases}1_{A_{i}} & \text { for } i \in R, \\ p_{i} & \text { for } i \notin R .\end{cases}
$$

Then, an element of the set (3.3), a multimorphism, is a morphism

$$
f: A_{1} \otimes \cdots \otimes A_{n} \longrightarrow B
$$

satisfying the equation

$$
f \circ\left(p_{1} \otimes \cdots \otimes p_{n}\right)=p \circ f \circ \sum_{\varnothing \neq R \subseteq\langle n\rangle} \lambda^{(\# R-1)} R(1) \otimes \cdots \otimes R(n) .
$$

This is a case of a general process of obtaining a multicategory structure on a category by restriction along a functor into a monoidal category. The notion of monoid makes sense in any multicategory. We have the following special case. 
Definition 3.3. A $\lambda$-weighted Rota-Baxter monoid in $\mathscr{V}$ is an object $(A, p)$ of $[\Sigma \mathbb{N}, \mathscr{V}]$ (that is, $p: A \rightarrow A$ in $\mathscr{V})$ equipped with the structure of semigroup on $J^{\mathrm{op}}(A, p)$ in the monoidal category Gph $\mathscr{V}$ of Proposition 3.1, and a unit $\eta: I \rightarrow A$ for the underlying semigroup $A$ in $\mathscr{V}$.

This definition should make the calculation of free weighted Rota-Baxter monoids possible; compare $[3,5,10,21]$.

To make Definition 3.3 a little more explicit, as expected, a $\lambda$-weighted Rota-Baxter monoid $(A, p)$ is a monoid $A$ in $\mathscr{V}$ equipped with an endomorphism $p: A \rightarrow A$ satisfying

$$
\mu \circ(p \otimes p)=p \circ \mu \circ(\lambda 1 \otimes 1+1 \otimes p+p \otimes 1) .
$$

Derivations and Rota-Baxter operators are not the only sources of semigroups and monoids for the monoidal structure of Proposition 3.1. The forgetful functor

$$
\text { ve: } \operatorname{Gph} \mathscr{V} \longrightarrow \mathscr{V} \times \mathscr{V}
$$

taking the graph $(A, E)$ to the pair $(A, E)$, is strict monoidal and has a right adjoint $\mathrm{R}$ defined by

$$
\mathrm{R}(X, Y)=(X, X \oplus X \oplus Y)
$$

with $s=\operatorname{pr}_{1}$ (the first projection) and $t=\mathrm{pr}_{2}$ (the second projection). It follows that $\mathrm{R}$ is monoidal and hence takes monoids to monoids.

Example 3.4. Take $\mathscr{V}=\operatorname{Mod}_{k}$, the category of modules over a commutative ring $k$. For a graph $(A, E)$ in this $\mathscr{V}$, we can write $e: a \rightarrow b$ to mean $a, b \in A, e \in E$ with $s(e)=a, t(e)=b$. For $k$ algebras $A$ and $B$, we obtain a monoid $\mathrm{R}(A, B)$ in Gph $\mathscr{V}:$ the graph is $\mathrm{pr}_{1}, \mathrm{pr}_{2}: A \oplus A \oplus B \rightarrow A$ and the multiplication is defined by:

$$
\left(\left(a_{1}, a_{2}, b\right): a_{1} \rightarrow a_{2}\right) \cdot\left(\left(c_{1}, c_{2}, d\right): c_{1} \rightarrow c_{2}\right)=\left(\lambda a_{1} c_{1}+a_{1} c_{2}+a_{2} c_{1}, a_{2} c_{2}, b d\right): a_{1} c_{1} \rightarrow a_{2} c_{2} .
$$

Of course the $\mathscr{V}$-functor $J(3.2)$ has both adjoints if $\mathscr{V}$ is complete and cocomplete enough. In particular, the right adjoint

$$
K: \quad \operatorname{Gph} \mathscr{V} \longrightarrow[\Sigma \mathbb{N}, \mathscr{V}]
$$

is defined by taking $K(A, E)$ to be the equalizer of the two morphisms

$$
s^{\mathbb{N}}, t^{\text {succ }}: E^{\mathbb{N}} \rightarrow A^{\mathbb{N}}
$$

equipped with the endomorphism $e: K(A, E) \rightarrow K(A, E)$ induced by $E^{\text {succ }}$. Here succ: $\mathbb{N} \rightarrow \mathbb{N}$ is the successor function $n \mapsto n+1$. Since $J$ (3.2) is strong monoidal for the monoidal structures under discussion, the adjunction $J \dashv K$ is monoidal. So $K$ takes semigroups to semigroups and monoids to monoids.

In particular, if $(A, p)$ is a $\lambda$-weighted Rota-Baxter monoid in $\mathscr{V}$, then $K$ takes the graph $(A, A)$ with $s=1_{A}$ and $t=p$ to a $\lambda$-weighted derivational semigroup in $\mathscr{V}$. The underlying object is the limit of the diagram

$$
A \stackrel{p}{\longleftarrow} A \stackrel{p}{\longleftarrow} A \stackrel{p}{\longleftarrow} \cdots
$$

in $\mathscr{V}$. 
Example 3.5. Taking $\mathscr{V}=$ Vect $_{k}$ and a $\lambda$-weighted Rota-Baxter $k$-algebra $p: A \rightarrow A$, we have the non-unital $\lambda$-weighted derivational $k$-algebra

$$
K\left(J(A, p)^{\mathrm{op}}\right)=\left\{a \in A^{\mathbb{N}} \mid p\left(a_{n+1}\right)=a_{n}\right\}
$$

with $d(a)_{n}=a_{n+1}$. The multiplication on $K\left(J(A, p)^{\text {op }}\right)$ is the restriction of the $\lambda$-weighted Hurwitz multiplication on $A^{\mathbb{N}}$ arising from the non-unital algebra $(A, \diamond)$ of Proposition 2.10. Moreover, $K\left(J(A, p)^{\text {op }}\right)$ supports a $\lambda$-weighted Rota-Baxter operator $p$ defined by $p(a)_{n}=$ $p\left(a_{n}\right)$. Notice too that $d \circ p=1$.

We conclude this section by describing the promonoidal structure in the sense of Day [8] with respect to which the monoidal structure of Proposition 3.1 is convolution.

Let $\mathbb{G}$ denote the category whose only objects are 0 and 1 , with the only non-identity morphisms $\sigma, \tau: 1 \rightarrow 0$. Write $I_{*} \mathbb{G}$ for the free $\mathscr{V}$-category on $\mathscr{V}$. Then Gph $\mathscr{V}=[\mathbb{G}, \mathscr{V}]=\left[I_{*} \mathbb{G}, \mathscr{V}\right]$ where the first set of square brackets means the ordinary functor category while the second means the $\mathscr{V}$-enriched functor category. The promonoidal structure in question is technically on $I_{*} \mathbb{G}$ in the $\mathscr{V}$-enriched sense. However, we can look at it as consisting of an ordinary a functor

$$
\mathrm{P}: \mathbb{G}^{\mathrm{op}} \times \mathbb{G}^{\mathrm{op}} \longrightarrow \mathrm{Gph} \mathscr{V}
$$

and an object $\mathrm{J} \in \mathrm{Gph} \mathscr{V}$. Of course $\mathrm{J}$ is just the graph $0,1: I \rightarrow I$ which is the tensor unit. We can regard P as a "cograph of cographs of graphs" (although a cograph looks just like a graph):

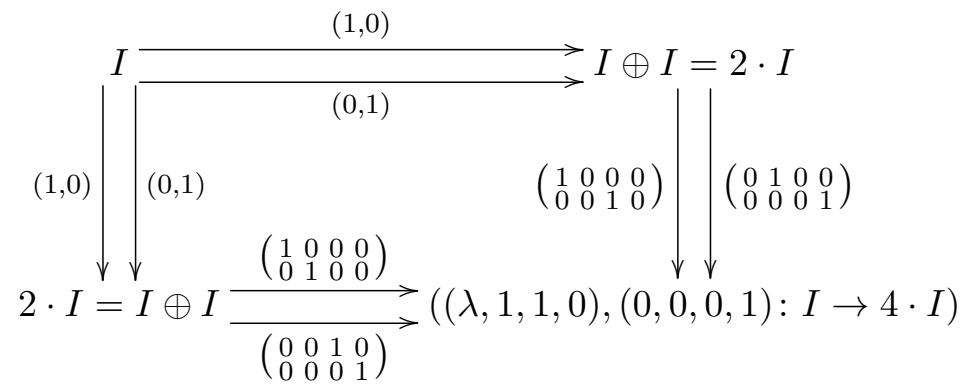

\section{The $L$-Hurwitz product of species}

Let $\mathfrak{S}$ denote the groupoid whose objects are finite sets and whose morphisms are bijective functions. We write $U+V$ for the disjoint union of sets $U$ and $V$; this is the binary coproduct as objects of the category Set of sets and all functions. It is not the coproduct in $\mathfrak{S}$; yet it does provide the symmetric monoidal structure on $\mathfrak{S}$ of interest here. When we write $X=A+B$ for $A$ and $B$ subsets of a set $X$, we mean $X=A \cup B$ and $\varnothing=A \cap B$.

We have the particular finite sets $\langle n\rangle=\{1,2, \ldots, n\}$.

Let $\mathscr{V}$ denote a monoidal category with finite coproducts which are preserved by tensoring on either side by an object. The tensor product of $V, W \in \mathscr{V}$ is denoted by $V \otimes W$ and the unit object by $I$. Justified by coherence theorems (see [16] for example), we write as if the monoidal structure on $\mathscr{V}$ were strictly associative and strictly unital. For any set $S$, write $S \cdot V$ for the coproduct of $S$ copies of $V \in \mathscr{V}$, when it exists (as it does for $S$ finite).

The category of $\mathscr{V}$-valued Joyal species, after [13, 14], is the functor category [S, $\mathscr{V}]$. The objects will simply be called species when $\mathscr{V}$ is understood.

Suppose $L: \mathfrak{S} \rightarrow \mathcal{Z} \mathscr{V}$ is a braided strong monoidal functor into the monoidal centre (in the sense of [15]) of $\mathscr{V}$. We have natural isomorphisms

$$
u_{X, V}: L X \otimes V \cong V \otimes L X,
$$


such that

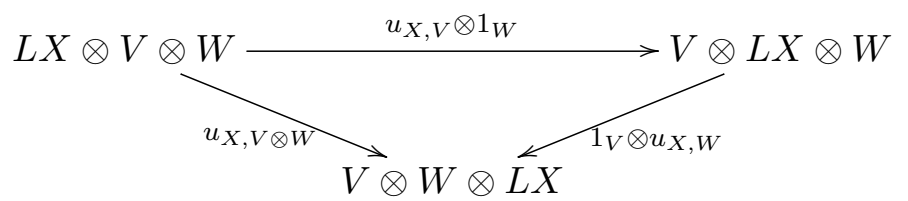

If $\mathscr{V}$ itself is braided (a fortiori symmetric), we can take a braided strong monoidal functor $\mathfrak{S} \rightarrow \mathscr{V}$ and compose it with the canonical braided strong monoidal functor $\mathscr{V} \rightarrow \mathcal{Z} \mathscr{V}$ to obtain such an $L$.

By way of example of an $L: \mathfrak{S} \rightarrow \mathcal{Z} \mathscr{V}$, we could take any finite set $\Lambda$ and $L X=\Lambda^{X} \cdot I$ with $L \sigma=\Lambda^{\sigma^{-1}} \cdot I$ for any bijective function $\sigma$.

Definition 4.1. The $L$-Hurwitz product $F \otimes^{L} G$ of species $F$ and $G$ is defined on objects $X \in \mathfrak{S}$ by

$$
\left(F \otimes L{ }^{L} G\right) X=\sum_{X=U \cup V} L(U \cap V) \otimes F U \otimes G V
$$

The definition of $F \otimes^{L} G$ on morphisms is clear since any bijective function $\sigma: X \rightarrow Y$ restricts to bijections

$$
U \rightarrow \sigma U, \quad V \rightarrow \sigma V, \quad U \cup V \rightarrow \sigma U \cup \sigma V, \quad U \cap V \rightarrow \sigma U \cap \sigma V .
$$

Let $J: \mathfrak{S} \rightarrow \mathscr{V}$ be the species whose value at $X$ is the unit $I$ for tensor in $\mathscr{V}$ when $X$ is empty and is initial in $\mathscr{V}$ otherwise. Clearly $J$ is a unit for the $L$-Hurwitz product in the sense that we have canonical isomorphisms

$$
\lambda_{G}: J \otimes^{L} G \rightarrow G \quad \text { and } \quad \rho_{F}: F \rightarrow F \otimes^{L} J .
$$

Associativity isomorphisms

$$
\alpha_{F, G, H}:\left(F \otimes^{L} G\right) \otimes^{L} H \cong F \otimes^{L}\left(G \otimes^{L} H\right)
$$

are obtained using the following result easily proved by Venn diagrams.

Lemma 4.2. $(U \cup V) \cap W+U \cap V \cong U \cap(V \cup W)+V \cap W$.

Then, to define (4.3), we use the isomorphisms

$$
\begin{aligned}
L((U & \cup V) \cap W) \otimes L(U \cap V) \otimes F U \otimes G V \otimes H W \\
& \cong L((U \cup V) \cap W+U \cap V) \otimes F U \otimes G V \otimes H W \\
& \cong L(U \cap(V \cup W)+V \cap W) \otimes F U \otimes G V \otimes H W \\
& \cong L(U \cap(V \cup W)) \otimes L(V \cap W) \otimes F U \otimes G V \otimes H W \\
& \cong L(U \cap(V \cup W)) \otimes F U \otimes L(V \cap W) \otimes G V \otimes H W,
\end{aligned}
$$

the first and third coming from the strong monoidal structure on $L$, the second from Lemma 4.2, and the fourth from the monoidal centre structure (4.1) on $L(V \cap W)$.

In the case $L=J$, we recover from (4.2) the usual convolution (Cauchy) product of species appearing in [13]. In the case where $L$ is the exponential series $L X=I$ for all $X$, we recover the Heisenberg product appearing in [1, 20].

For a general $L$, the term $L(U \cap V)$ can be considered a measure of the failure of $U$ and $V$ to be disjoint. 


\section{A combinatorial interpretation}

We consider the case where $\mathscr{V}=$ Set so that [ $\mathfrak{S}$, Set] is the category of species as studied in [13]. Fix any set $\Lambda$. Define the species $L$ by

$$
L X=\left\{S=\left(S_{\lambda}\right)_{\lambda \in \Lambda} \mid S_{\lambda} \subseteq X, \sum_{\lambda \in \Lambda} S_{\lambda}=X\right\}
$$

and $(L \sigma) S=\left(\sigma S_{\lambda}\right)_{\lambda \in \Lambda}$. In other words, a structure of the species $L$ on the set $X$ is a partition of $X$ into a $\Lambda$-indexed family of disjoint (possibly empty) subsets.

A structure of the species $F \otimes{ }^{L} G$ on the set consists of a quintuplet $(U, V, S, \phi, \gamma)$ where $U, V$ are subsets of $X$ such that $X=U \cup V$, and $S, \phi, \gamma$ are $L-, F$-, G-structures on $U \cap V, U, V$, respectively.

We write $\# S$ for the cardinality of the set $S$. We assume $\Lambda$ is finite and put $\lambda=\# \Lambda$.

The cardinality sequence of a species $F$ is the sequence $\# F: \mathbb{N} \rightarrow \mathbb{Z}$ defined by

$$
(\# F)(n)=\# F\langle n\rangle \text {. }
$$

We consider the $\lambda$-Hurwitz product $(2.2)$ on $\mathbb{Z}^{\mathbb{N}}$.

Proposition 5.1. \# $\left(F \otimes^{L} G\right)=\# F \cdot{ }^{\lambda} \# G$.

This result specializes to Theorem 2.7 of [1] when $L$ is the exponential species and $\lambda=1$.

\section{The iterated tensor and coherence}

Proposition 6.1. An alternative definition of $F \otimes{ }^{L} G$ is

$$
\left(F \otimes L{ }^{L} G\right) X=\sum_{X=A+B+C} L(C) \otimes F(A+C) \otimes G(B+C) .
$$

Proof. Given $X=A+B+C$, put $U=A+C$ and $V=B+C$. Given $X=U \cup V$, put $A=U \backslash V, B=V \backslash U$, and $C=U \cap V$.

The $n$-fold version of this tensor product is

$$
\begin{aligned}
& \otimes_{n}^{L}\left(F_{1}, \ldots, F_{n}\right) X \\
& \quad=\sum_{X=\sum_{\varnothing \neq S \subseteq\langle n\rangle} A_{S}} L\left(\sum_{S}(\# S-1) \cdot A_{S}\right) \otimes F_{1}\left(\sum_{1 \in S} A_{S}\right) \otimes \cdots \otimes F_{n}\left(\sum_{n \in S} A_{S}\right) .
\end{aligned}
$$

This yields the formula in Proposition 6.1 for $n=2$ by taking $A=A_{1}, B=A_{2}, C=A_{\{1,2\}}$. Note that (6.1) is unchanged if we replace $\langle n\rangle$ by any set of cardinality $n$.

Remark 6.2. As Joachim Kock reminded me, if we replace $\langle n\rangle$ by the ' $(n-1)$-simplex' $[n-1]=$ $\{0,1, \ldots, n-1\}$, then the non-empty subsets $S$ correspond to the non-degenerate faces of $[n-1]$ and $\# S-1$ is the dimension of the face.

Let us consider the effect of inserting one pair of parentheses in a multiple tensor (6.1). We look at

$$
\otimes_{p+1+r}^{L}\left(F_{1}, \ldots, F_{p}, \otimes_{q}^{L}\left(F_{p+1}, \ldots, F_{p+q}\right), F_{p+q+1}, \ldots, F_{p+q+r}\right) X .
$$


Using (6.1) twice, once with $n=p+1+r$ and once with $n=q$, we obtain the expression

$$
\begin{aligned}
& L\left(\sum_{T}(\# T-1) \cdot B_{T}\right) \otimes F_{1}\left(\sum_{1 \in T} B_{T}\right) \otimes \cdots \otimes F_{p}\left(\sum_{p \in T} B_{T}\right) \\
& \otimes L\left(\sum_{R}(\# R-1) \cdot C_{R}\right) \otimes F_{p+1}\left(\sum_{p+1 \in R} C_{R}\right) \otimes \cdots \otimes F_{p+q}\left(\sum_{p+q \in R} C_{R}\right) \\
& \otimes F_{p+q+1}\left(\sum_{p+q+1 \in T} B_{T}\right) \otimes \cdots \otimes F_{p+q+r}\left(\sum_{p+q+r \in T} B_{T}\right)
\end{aligned}
$$

summed over all families

$$
B=\left(B_{T} \mid \varnothing \neq T \subseteq\{1, \ldots, p, \star, p+q+1, \ldots, p+q+r\}\right)
$$

providing a partition $X=\sum_{T} B_{T}$ of $X$, together with all families

$$
C=\left(C_{R} \mid \varnothing \neq R \subseteq\{p+1, \ldots, p+q\}\right)
$$

providing a partition $\sum_{\star \in T} B_{T}=\sum_{R} C_{R}$ of $\sum_{\star \in T}$. Using the fact that $L$ lands in $\mathcal{Z} \mathscr{V}$ and that $L$ is strong monoidal, we obtain the isomorphic expression

$$
\begin{gathered}
L\left(\sum_{T}(\# T-1) \cdot B_{T}+\sum_{R}(\# R-1) \cdot C_{R}\right) \otimes F_{1}\left(\sum_{1 \in T} B_{T}\right) \otimes \cdots \otimes F_{p}\left(\sum_{p \in T} B_{T}\right) \\
\otimes F_{p+1}\left(\sum_{p+1 \in R} C_{R}\right) \otimes \cdots \otimes F_{p+q}\left(\sum_{p+q \in R} C_{R}\right) \\
\otimes F_{p+q+1}\left(\sum_{p+q+1 \in T} B_{T}\right) \otimes \cdots \otimes F_{p+q+r}\left(\sum_{p+q+r \in T} B_{T}\right)
\end{gathered}
$$

summed over the same families $(B, C)$. For $\star \in T$, we have $B_{T}=\sum_{R} C_{R} \cap B_{T}$. On the other hand, $C_{R}=\sum_{\star \in T} C_{R} \cap B_{T}$. Put

$$
Q=\{p+1, \ldots, p+q\} \quad \text { and } \quad N=\{1, \ldots, p\} \cup\{p+q+1, \ldots, p+q+r\},
$$

and obtain a family

$$
A=\left(A_{S} \mid \varnothing \neq S \subseteq\langle p+q+r\rangle\right)
$$

partitioning $X$ by defining

$$
A_{S}= \begin{cases}B_{S} & \text { for } S \cap Q=\varnothing, \\ C_{S \cap Q} \cap B_{(S \cap N) \cup\{\star\}} & \text { for } S \cap Q \neq \varnothing .\end{cases}
$$

Then we can recover the $B$ and $C$ families via

$$
B_{T}=\left\{\begin{array}{ll}
A_{T} & \text { for } \star \notin T, \\
\sum_{R} A_{R \cup(T \backslash \star)} & \text { for } \star \in T,
\end{array} \quad \text { and } \quad C_{R}=\sum_{\star \in T} A_{R \cup(T \backslash \star)} .\right.
$$


We have the following equations

(i)

$$
\begin{aligned}
& \text { (i) } \quad \sum_{S}(\# S-1) \cdot A_{S}=\sum_{T}(\# T-1) \cdot B_{T}+\sum_{R}(\# R-1) \cdot C_{R}, \\
& \text { (ii) } \quad \sum_{k \in S} A_{S}= \begin{cases}\sum_{k \in T} B_{T} \quad \text { for } 1 \leq k \leq p \text { or } p+q+1 \leq k \leq p+q+r, \\
\sum_{k \in R} C_{R} & \text { for } p+1 \leq k \leq p+q .\end{cases}
\end{aligned}
$$

This shows that the sum of the expressions (6.2) over the pairs $(B, C)$ is equal to (6.1) with $n=p+q+r$. Remember however that the tensor product + on $\mathfrak{S}$ is not strict symmetric; the symmetry on $\mathfrak{S}$ provides canonical bijections between the left- and right-hand sides of (i) and (ii). Since $L$ is braided, we have constructed a natural isomorphism

$$
\begin{aligned}
a_{p, q, r}: & \otimes_{n}^{L}\left(F_{1}, \ldots, F_{p+q+r}\right) \\
& \cong \otimes_{p+1+r}^{L}\left(F_{1}, \ldots, F_{p}, \otimes_{q}^{L}\left(F_{p+1}, \ldots, F_{p+q}\right), F_{p+q+1}, \ldots, F_{p+q+r}\right) .
\end{aligned}
$$

Now consider the Mac Lane-Stasheff pentagon for 2-fold bracketings of $F_{1} \otimes{ }^{L} F_{2} \otimes{ }^{L} F_{3} \otimes{ }^{L} F_{4}$ as the vertices. Let $a: H \rightarrow K$ denote one of the edges of the pentagon obtained using the associativity isomorphisms (4.3). There is a composite $b$ of two isomorphisms, each using one instance of an isomorphism (6.3), which goes from $\otimes_{4}^{L}\left(F_{1}, F_{2}, F_{3}, F_{4}\right)$ to $H$, and another one $c: \otimes_{4}^{L}\left(F_{1}, F_{2}, F_{3}, F_{4}\right) \rightarrow H$. By coherence of the braided strong monoidal functor $L$, it follows that $a \circ b=c$. Commutativity of the pentagon is a consequence of commutativity of all these triangular sides of the so-formed pentagonal cone.

\section{$7 \quad$ Promonoidal structures on $\mathfrak{S}$}

For finite sets $A, B$ and $X$, let $\operatorname{Cov}(A, B ; X)$ denote the set of jointly surjective pairs $(\mu, \nu)$ of injective functions

$$
A \stackrel{\mu}{\longrightarrow} X \stackrel{\nu}{\longleftarrow} B \text {. }
$$

We write $A \times_{X} B$ for the pullback of $\mu$ and $\nu$.

Define a functor

$$
\mathrm{P}: \mathfrak{S}^{\mathrm{op}} \times \mathfrak{S}^{\mathrm{op}} \times \mathfrak{S} \longrightarrow \mathscr{V}
$$

by

$$
\mathrm{P}(A, B ; X)=\sum_{(\mu, \nu) \in \operatorname{Cov}(A, B ; X)} L\left(A \times_{X} B\right) .
$$

Proposition 7.1. $\left(F \otimes \otimes^{L} G\right) X \cong \int{ }^{A, B} \mathrm{P}(A, B ; X) \otimes F A \otimes G B$.

Proof. A universal dinatural transformation

$$
\theta_{A, B}: \mathrm{P}(A, B ; X) \otimes F A \otimes G B \longrightarrow \sum_{X=U \cup V} L(U \cap V) \otimes F U \otimes G V
$$

is defined by taking its composite with the injection at $(\mu, \nu) \in \operatorname{Cov}(A, B ; X)$ to be obtained from the $(\mu(A), \nu(B))$ injection and the bijections $A \cong \mu(A), B \cong \nu(B), A \times_{X} B \cong \mu(A) \cap \nu(B)$, noting $X=\mu(A) \cup \nu(B)$.

By Day's general theory of promonoidal categories $[7,8]$, we have

Corollary 7.2. If moreover $\mathscr{V}$ is (left and right) closed and sufficiently complete then $\otimes^{L}$ defines $a$ (left and right) closed monoidal structure on [S, $\mathscr{V}]$. The monoidal structure coincides with that of Section 4. 


\section{The weighted bimonoidale structure on fam $\mathfrak{S}$}

Lately (for example, in [6]), we have used the term monoidale for "pseudomonoid", also called "monoidal object", in a monoidal bicategory $\mathscr{M}[9]$. For example, the monoidales in the cartesian monoidal bicategory Cat are monoidal categories.

When the monoidal bicategory $\mathscr{M}$ is symmetric, the monoidales themselves form a symmetric monoidal bicategory where the morphisms are strong monoidal. With the same tensor product, the opposite bicategory $\mathscr{M}^{\text {op }}$ is symmetric monoidal. A bimonoidale in $\mathscr{M}$ is a monoidale in $\mathscr{M}^{\mathrm{op}}$. Incidentally, every monoidale in Cat is uniquely a bimonoidale.

Consider the 2-category Cat + of (small) categories admitting finite coproducts, and finitecoproduct-preserving functors. This becomes a symmetric closed monoidal bicategory (see [9]) with tensor product $\mathscr{A} \otimes \mathscr{B}$ representing functors $H: \mathscr{A} \times \mathscr{B} \rightarrow \mathscr{X}$ for which each $H(A,-)$ and each $H(-, B)$ is finite coproduct preserving. Clearly the monoidal category $\mathscr{V}$ of Section 4 is a monoidale (= pseudomonoid) in $\mathrm{Cat}_{+}$.

For any category $\mathscr{C}$, we write fam $\mathscr{C}$ for the free finite coproduct completion of $\mathscr{C}$. That is, fam provides the left biadjoint to the forgetful 2-functor Cat $\rightarrow$ Cat. Indeed, fam is a strong monoidal pseudofunctor; in particular, there is a canonical equivalence

$\operatorname{fam} \mathscr{C} \otimes \operatorname{fam} \mathscr{D} \simeq \operatorname{fam}(\mathscr{C} \times \mathscr{D})$

Every monoidal category $\mathscr{C}$ determines a monoidale fam $\mathscr{C}$ in Cat .

Explicitly, the objects of fam $\mathscr{C}$ can be written formally as $\sum_{s \in S} C_{s}$ where $S$ is a finite set and $C_{s} \in \mathscr{C}$. Then, if $\mathscr{C}$ is monoidal, the monoidale structure on fam $\mathscr{C}$ is defined by

$$
\sum_{s \in S} C_{s} \otimes \sum_{t \in T} D_{t}=\sum_{(s, t) \in S \times T} C_{s} \otimes D_{t}
$$

We are interested in fam $\mathfrak{S}$. By what we have just said, this is a monoidale in Cat + :

$$
\sum_{s \in S} U_{s} \otimes \sum_{t \in T} V_{t}=\sum_{(s, t) \in S \times T}\left(U_{s}+V_{t}\right)
$$

Fix a finite set $\Lambda$ and define $L: \mathfrak{S} \rightarrow$ Set by $L X=\Lambda^{X}$ and $L \sigma=\Lambda^{\sigma^{-1}}$. Define a coproductpreserving functor

$$
\Delta: \operatorname{fam} \mathfrak{S} \longrightarrow \operatorname{fam}(\mathfrak{S} \times \mathfrak{S}) \simeq \operatorname{fam} \mathfrak{S} \otimes \text { fam } \mathfrak{S}
$$

by

$$
\Delta(X)=\sum_{X=A+B+C} L(C) \cdot(A+C, B+C)
$$

for $X \in \mathfrak{S}$.

Proposition 8.1. The functor $\Delta$ of (8.1) is strong monoidal.

Proof. In $\Delta(X+Y)=\sum_{X+Y=A+B+C} L(C) \cdot(A+C, B+C)$ we can put

$$
\begin{aligned}
& P=X \cap A, \quad Q=X \cap B, \quad R=X \cap C, \\
& U=Y \cap A, \quad V=Y \cap B, \quad W=Y \cap C
\end{aligned}
$$


to obtain

$$
\begin{aligned}
\Delta(X+Y) & =\sum_{X=P+Q+R, Y=U+V+W} L(R+W) \cdot(P+U+R+W, Q+V+R+W) \\
& \cong \sum_{X=P+Q+R} L(R) \cdot(P+R, Q+R) \times \sum_{Y=U+V+W} L(W) \cdot(U+W, V+W) \\
& \cong \Delta X \times \Delta Y,
\end{aligned}
$$

as required.

The relationship between this structure and the promonoidal structure of Section 7 will be examined elsewhere; indeed, see [11].

\section{Weighted categorical derivations}

Suppose $\mathscr{V}$ is a symmetric monoidal closed category which is complete and cocomplete, and suppose $L: \mathfrak{S} \rightarrow \mathscr{V}$ is a strong monoidal functor.

Harking back to Remark 2.11, we are prompted to consider the 2-category

$$
\mathfrak{E}=\operatorname{Hom}\left(\Sigma \mathfrak{S}, \mathscr{V}-\mathrm{Cat}_{L,+}\right) .
$$

Here $\Sigma \mathfrak{S}$ denotes the bicategory with one object (denoted $\star$ ) whose homcategory is the symmetric groupoid $\mathfrak{S}$; composition is provided by the monoidal structure + on $\mathfrak{S}$. Also $\mathscr{V}$-Cat $\mathrm{C}_{L,+}$ denotes the 2-category of $\mathscr{V}$-categories admitting finite coproducts and tensoring with the object $L(X)$ of $\mathscr{V}$; the morphisms are $\mathscr{V}$-functors preserving these colimits; the 2-cells are $\mathscr{V}$-natural transformations. The objects of (9.1) are pseudofunctors $T: \Sigma \mathfrak{S} \rightarrow \mathscr{V}$-Cat ${ }_{L,+}$, the morphisms are pseudonatural transformations, and the 2-cells are modifications (in terminology of [19]). Such an object $T$ determines a $\mathscr{V}$-category $T \star=\mathscr{M} \in \mathscr{V}$-Cat ${ }_{L,+}$ and a strong monoidal functor $T_{\star \star}: \mathfrak{S} \rightarrow \mathscr{V}$-Cat $_{L,+}(\mathscr{M}, \mathscr{M})$. This $T_{\star \star}$ is determined up to equivalence by an endomorphism $D: \mathscr{M} \rightarrow \mathscr{M}$ in $\mathscr{V}$-Cat ${ }_{L,+}$ and an involutive Yang-Baxter ${ }^{2}$ operator $\rho: D \circ D \Rightarrow D \circ D$ on $D$ (for example, see [15] for terminology). Then $T_{\star \star}\langle n\rangle \cong D^{\circ n}$ and, for the non-identity bijection $\tau:\langle 2\rangle \rightarrow\langle 2\rangle, T \tau$ transports to $\rho$. Therefore we shall write the object $T$ of $\mathfrak{E}$ (9.1) as a pair $\left(\mathscr{M}, D^{*}\right)$ where $T \star=\mathscr{M}$ and $T_{\star \star}=D^{*}$. The morphisms of $\mathfrak{E}$ are then squares

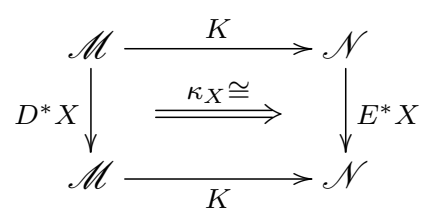

in $\mathscr{V}$-Cat ${ }_{L,+}$ which are $\mathscr{V}$-natural in $X$ and, stacking vertically, respect the tensor in $\mathfrak{S}$. Generalizing the tensor $\otimes$ on $\mathrm{Cat}_{+}$as in Section 8, we have a tensor, also denoted by $\nabla$, on $\mathscr{V}$-Cat ${ }_{L,+}$, where the tensor product $\mathscr{A} \otimes \mathscr{B}$ represents $\mathscr{V}$-functors $H: \mathscr{A} \otimes \mathscr{B} \rightarrow \mathscr{X}$ for which each of $H(A,-)$ and $H(-, B)$ preserves finite coproducts and tensoring with each $L(X)$. This makes $\mathscr{V}$-Cat $L,+$ into a monoidal bicategory.

This tensor product $\otimes$ on $\mathscr{V}$-Cat ${ }_{L,+}$ lifts to one, denoted by $\widehat{\otimes}$, on $\mathfrak{E}(9.1)$ :

$$
\left(\mathscr{M}, D^{*}\right) \widehat{\otimes}\left(\mathscr{N}, E^{*}\right)=\left(\mathscr{M} \otimes \mathscr{N}, D^{*} \widehat{\otimes} E^{*}\right) m,
$$

where

$$
\left(D^{*} \widehat{\otimes} E^{*}\right) X=\sum_{X=U \cup V} L(U \cap V) \otimes D^{*} U \otimes E^{*} V .
$$

\footnotetext{
${ }^{2}$ This is Rodney Baxter http://en.wikipedia.org/wiki/Rodney_Baxter, not the author of [4].
} 
To see that $D^{*} \widehat{\nabla} E^{*}: \mathfrak{S} \rightarrow \mathscr{V}-\operatorname{Cat}_{L,+}(\mathscr{M} \otimes \mathscr{N}, \mathscr{M} \otimes \mathscr{N})$ is strong monoidal, we calculate

$$
\begin{aligned}
& \left(D^{*} \widehat{\otimes} E^{*}\right)(X+Y) \cong \sum_{X+Y=U \cup V} L(U \cap V) \otimes D^{*} U \otimes E^{*} V \\
& \cong \sum_{X=U_{1} \cup V_{1}, Y=U_{2} \cup V_{2}} L\left(U_{1} \cap V_{1}+U_{2} \cap V_{2}\right) \otimes\left(D^{*} U_{1} \circ D^{*} U_{2}\right) \otimes\left(E^{*} V_{1} \circ E^{*} V_{2}\right) \\
& \cong \sum_{X=U_{1} \cup V_{1}, Y=U_{2} \cup V_{2}} L\left(U_{1} \cap V_{1}\right) \otimes L\left(U_{2} \cap V_{2}\right) \otimes\left(D^{*} U_{1} \otimes E^{*} V_{1}\right) \circ\left(D^{*} U_{2} \otimes E^{*} V_{2}\right) \\
& \cong \sum_{X=U_{1} \cup V_{1}, Y=U_{2} \cup V_{2}} L\left(U_{1} \cap V_{1}\right) \otimes\left(D^{*} U_{1} \otimes E^{*} V_{1}\right) \circ L\left(U_{2} \cap V_{2}\right) \otimes\left(D^{*} U_{2} \otimes E^{*} V_{2}\right) \\
& \cong\left(D^{*} \widehat{\otimes} E^{*}\right) X \circ\left(D^{*} \widehat{\otimes} E^{*}\right) Y .
\end{aligned}
$$

In this way, $\mathfrak{E}(9.1)$ becomes a monoidal bicategory.

Definition 9.1. An $L$-weighted derivation $D^{*}$ on a monoidale $\mathscr{M}$ in $\mathscr{V}$-Cat ${ }_{L,+}$ is a lifting of the monoidale structure on $\mathscr{M}$ to a monoidale structure on $\left(\mathscr{M}, D^{*}\right)$ in $\mathfrak{E}(9.1)$.

Example 9.2. An $L$-weighted derivation $D^{*}: \mathfrak{S} \rightarrow \mathscr{V}-\mathrm{Cat}_{L,+}([\mathfrak{S}, \mathscr{V}],[\mathfrak{S}, \mathscr{V}])$ on the monoidale $\left([\mathfrak{S}, \mathscr{V}], \otimes^{L}\right)$ is defined by $\left(D^{*} X\right) F=F(X+-)$. The main point is the canonical isomorphism below

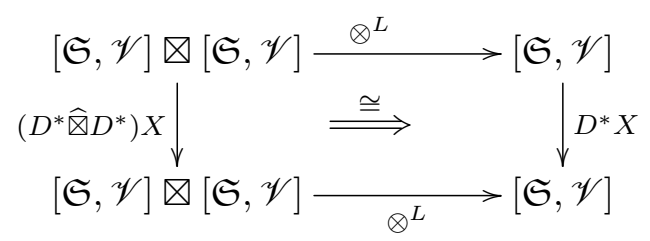

Remark 9.3. The first item of Remark 2.11 has a categorical version. The forgetful 2 -functor $\mathrm{U}: \mathfrak{E} \rightarrow \mathscr{V}-\mathrm{Cat}_{L,+}$ has a right biadjoint JS taking the $\mathscr{V}$-category $\mathscr{A}$ to the object of $\mathfrak{E}$ determined be the $\mathscr{V}$-category JS $\mathscr{A}=[\mathfrak{S}, \mathscr{A}]$ of species in $\mathscr{A}$, equipped with $L$-weighted derivation the $D^{*}$ just as in Example 9.2 with the codomain $\mathscr{V}$ replaced by $\mathscr{A}$. Since $\mathrm{U}$ is strong monoidal, the biadjunction $\mathrm{U} \dashv_{\mathrm{bi}} \mathrm{JS}$ is monoidal. Consequently the biadjunction lifts to one between the 2-categories of monoidales in $\mathfrak{E}$ and $\mathscr{V}$-Cat ${ }_{L,+}$. Indeed $\mathrm{U}$ is pseudocomonadic.

\section{The iterated tensor product again}

Observe the following simple reindexing of (4.2).

Proposition 10.1. An alternative definition of $F \otimes^{L} G$ is

$$
\left(F \otimes{ }^{L} G\right) X=\sum_{V \subseteq U \subseteq X} L(U \backslash V) \otimes F(U) \otimes G(X \backslash V) .
$$

This leads us to another formula for the $n$-fold $L$-weighted tensor product. Define the modified $n$-filtration set $^{3}$ on any finite set $X$ by

$$
\begin{aligned}
\operatorname{mFil}_{n} X=\{ & (U, V) \mid U=\left(0=U_{0} \subseteq U_{1} \subseteq \cdots \subseteq U_{n-1} \subseteq U_{n}=X\right), \\
& \left.V=\left(V_{0}, V_{1}, \ldots, V_{n-1}\right) \text { with } V_{i} \subseteq U_{i} \text { for } 0 \leq i<n\right\}
\end{aligned}
$$

\footnotetext{
${ }^{3}$ We are "modifying" the filtration $U$ of $X$ by equipping it with the extra subsets $V$.
} 
Proposition 10.2. An alternative definition of the $n$-fold tensor product (6.1) is

$$
\begin{aligned}
\otimes_{n}^{L}\left(F_{1}, \ldots, F_{n}\right) X= & \sum_{(U, V) \in \mathrm{mFil}_{n} X} L\left(U_{1} \backslash V_{1}\right) \otimes \cdots \otimes L\left(U_{n-1} \backslash V_{n-1}\right) \\
& \otimes F_{1}\left(U_{1} \backslash V_{0}\right) \otimes \cdots \otimes F_{n}\left(U_{n} \backslash V_{n-1}\right) .
\end{aligned}
$$

Proof. The formula follows by repeated application of the formula of Proposition 10.1 in evaluating the left bracketing

$$
\left(\cdots\left(F_{1} \otimes^{L} F_{2}\right) \otimes^{L} \ldots\right) \otimes^{L} F_{n}
$$

at $X$.

Let us relate the formulas (6.1) and (10.1) in the case $n=3$. A modified 3-filtration $(U, V) \in \mathrm{mFil}_{3} X$ of $X$ amounts to subsets $U_{1} \subseteq U_{2} \subseteq X$ and $V_{1} \subseteq U_{1}, V_{2} \subseteq U_{2}$. With this we can define

$$
\begin{array}{ll}
A_{1}=V_{1} \cap V_{2}, \quad A_{2}=V_{2} \backslash U_{1} \cap V_{2}, & A_{3}=X \backslash U_{2}, \\
A_{12}=U_{1} \cap V_{2} \backslash A_{1}, \quad A_{13}=V_{1} \backslash A_{1}, & A_{23}=\left(U_{2} \backslash U_{1}\right) \backslash A_{2}, \quad A_{123}=\left(U_{1} \backslash V_{1}\right) \backslash A_{12}
\end{array}
$$

and verify that $X=A_{1}+A_{2}+A_{3}+A_{12}+A_{13}+A_{23}+A_{123}$. Conversely, given the partition $A$ of $X$, we can define

$$
U_{1}=X \backslash\left(A_{2}+A_{3}+A_{23}\right), \quad U_{2}=X \backslash A_{3}, \quad V_{1}=A_{1}+A_{13}, \quad V_{2}=A_{1}+A_{2}+A_{12} .
$$

\section{Tensor products for charades}

The term "charade" is intended in the sense of Kapranov [18, Definition 3.2] and is related to Hall algebras (for example, see [22, Section 2]). One has a small abelian (or triangulated) category $\mathscr{A}$ and looks at functors defined on the groupoid $\mathscr{A}_{\mathrm{g}}$ of invertible morphisms in $\mathscr{A}$. A promonoidal structure is defined on $\mathscr{A}_{\mathrm{g}}$ using the short exact sequences (or triangles) of $\mathscr{A}$. The functors are tensored using convolution. Here we will only discuss the case where $\mathscr{A}$ is the category of finite vector spaces over a fixed finite field $\mathbb{F}_{q}$. We make a conjecture that a family of weighted monoidal structures exists and give some evidence for it.

Motivated by Proposition 10.1, we consider generalizing the tensor product of [17]. Let $\mathfrak{G}_{q}$ be the groupoid of finite vector spaces over the field $\mathbb{F}_{q}$ of cardinality $q$; the morphisms are linear bijections. We write $V \leq U$ to mean $V$ is an $\mathbb{F}_{q}$-linear subspace of $U$, and we write $U / V$ for the quotient space.

To be specific, take $\mathscr{V}=$ Vect $_{\mathbb{C}}$ to be the category of complex vector spaces with all linear functions.

Let $L: \mathfrak{G}_{q} \rightarrow \mathscr{V}$ be a suitable functor: we will consider conditions on it later.

For functors $F, G: \mathfrak{G}_{q} \rightarrow \mathscr{V}$, define $F \otimes^{L} G: \mathfrak{G}_{q} \rightarrow \mathscr{V}$ by

$$
\left(F \otimes L{ }^{L} G\right) X=\sum_{V \leq U \leq X} L(U / V) \otimes F(U) \otimes G(X / V) .
$$

This leads us to an $n$-fold tensor product in a manner analogous to (10.1). Define the modified $n$-flag set on any finite $\mathbb{F}_{q}$-vector space $X$ by

$$
\begin{array}{r}
\operatorname{mFlg}_{n} X=\left\{(U, V) \mid U=\left(0=U_{0} \leq U_{1} \leq \cdots \leq U_{n-1} \leq U_{n}=X\right),\right. \\
\left.V=\left(V_{0}, V_{1}, \ldots, V_{n-1}\right) \text { with } V_{i} \leq U_{i} \text { for } 0 \leq i<n\right\}
\end{array}
$$


Now we put

$$
\begin{aligned}
\otimes_{n}^{L}\left(F_{1}, \ldots, F_{n}\right) X= & \sum_{(U, V) \in \mathrm{mFlg} n} L\left(U_{1} \backslash V_{1}\right) \otimes \cdots \otimes L\left(U_{n-1} \backslash V_{n-1}\right) \\
& \otimes F_{1}\left(U_{1} \backslash V_{0}\right) \otimes \cdots \otimes F_{n}\left(U_{n} \backslash V_{n-1}\right) .
\end{aligned}
$$

The formula follows by repeated application of (11.1) in evaluating the left bracketing

$$
\left(\cdots\left(F_{1} \otimes^{L} F_{2}\right) \otimes^{L} \ldots\right) \otimes^{L} F_{n}
$$

at $X$.

Let us look at the ternary tensor product

$$
\otimes_{3}^{L}(F, G, H) X=\left(\left(F \otimes^{L} G\right) \otimes^{L} H\right) X
$$

It is a direct sum over modified 3-flags $(U, V)$ on $X$; that is, subspaces $U_{1} \leq U_{2} \leq X, V_{1} \leq U_{1}$ and $V_{2} \leq U_{2}$. From these we can uniquely define vector spaces $A_{S}$ for each $\varnothing \neq S \subseteq\langle 3\rangle$ via the following diagrams of short exact sequences:
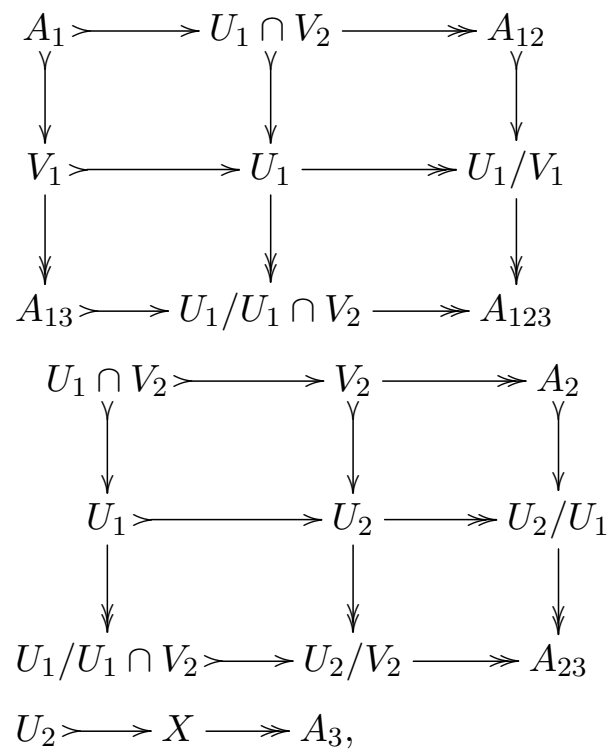

from which we see

$$
X \cong A_{1} \oplus A_{2} \oplus A_{3} \oplus A_{12} \oplus A_{23} \oplus A_{13} \oplus A_{123}
$$

Note also the isomorphisms

$$
\begin{aligned}
& U_{1} / V_{1} \cong A_{12} \oplus A_{123}, \quad U_{2} / V_{2} \cong A_{13} \oplus A_{23} \oplus A_{123}, \\
& U_{1} \cong A_{1} \oplus A_{12} \oplus A_{13} \oplus A_{123}, \quad U_{2} / V_{1} \cong A_{2} \oplus A_{12} \oplus A_{23} \oplus A_{123}, \\
& X / V_{2} \cong A_{3} \oplus A_{13} \oplus A_{23} \oplus A_{123} .
\end{aligned}
$$

On the other hand, we can see that the formula for the right bracketing is

$$
\left(F \otimes^{L}\left(G \otimes^{L} H\right)\right) X=\sum_{M \leq N \leq X, M \leq I \leq J \leq X} L(N / M) \otimes L(J / I) \otimes F N \otimes G(J / M) \otimes H(X / I) .
$$


We can see that this indexing set also leads to a direct sum decomposition (11.2) from the following diagrams of short exact sequences:

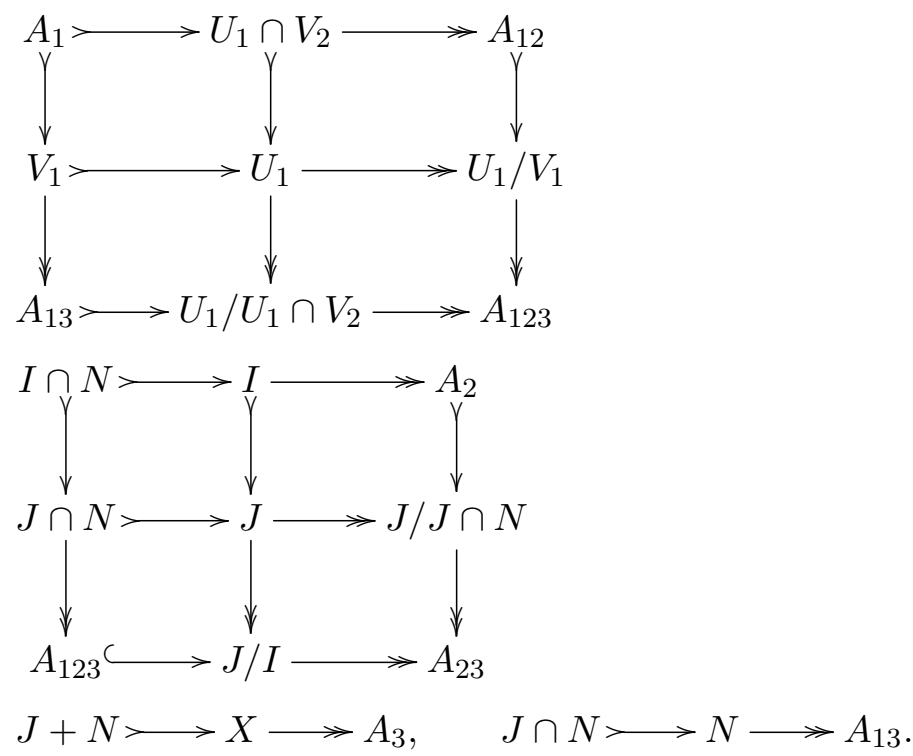

Note also the isomorphisms

$$
\begin{aligned}
& N / M \cong A_{12} \oplus A_{13} \oplus A_{123}, \quad J / I \cong A_{23} \oplus A_{123}, \quad N \cong A_{1} \oplus A_{12} \oplus A_{13} \oplus A_{123}, \\
& J / M \cong A_{2} \oplus A_{12} \oplus A_{23} \oplus A_{123}, \quad X / I \cong A_{3} \oplus A_{13} \oplus A_{23} \oplus A_{123} .
\end{aligned}
$$

In order to have an associativity isomorphism we at least need a canonical isomorphism

$$
L\left(A_{12} \oplus A_{123}\right) \otimes L\left(A_{13} \oplus A_{23} \oplus A_{123}\right) \cong L\left(A_{12} \oplus A_{13} \oplus A_{123}\right) \otimes L\left(A_{23} \oplus A_{123}\right) .
$$

We do have such an isomorphism if $L: \mathfrak{G}_{q} \rightarrow \mathscr{V}$ takes direct sums to tensor products; of course, direct sum of $\mathbb{F}_{q}$-vector spaces is neither product nor coproduct in $\mathfrak{G}_{q}$. This is still merely evidence that the desired associativity isomorphism should exist: it is not a complete definition.

Recall from [17] that $\mathfrak{G}_{q}$ has a braided promonoidal structure. The convolution structure on $\left[\mathfrak{G}_{q}, \mathscr{V}\right]$ arising from this (as per Day $[7,8]$ ) is precisely the tensor product $F \otimes^{J} G$ where $J X=I$ for $X=0$ and $J X=0$ for $X \neq 0$.

Conjecture 11.1. If $L$ is braided strong promonoidal then (11.1) defines a monoidal structure $\otimes^{L}$ on $\left[\mathfrak{G}_{q}, \mathscr{V}\right]$.

Should this be the case, the tensor $\otimes^{L}$ on $\left[\mathfrak{G}_{q}, \mathscr{V}\right]$ would be obtained from quite an interesting promonoidal structure on $\mathfrak{G}_{q}$. A short sequence

$$
A>\stackrel{f}{\longrightarrow} X \stackrel{g}{\longrightarrow} B
$$

in Vect $_{\mathbb{F}_{q}}$ might be called short pre-exact when $f$ is a monomorphism, $g$ is an epimorphism and $\operatorname{ker} g \leq \operatorname{im} f$. Write $\operatorname{Spes}(A, B ; X)$ for the set of such $(f, g)$. Put

$$
\mathrm{P}(A, B ; X)=\sum_{(f, g) \in \operatorname{Spes}(A, B ; X)} L(\operatorname{im}(g \circ f)) .
$$

This $\mathrm{P}: \mathfrak{G}_{q}^{\mathrm{op}} \times \mathfrak{G}_{q}^{\mathrm{op}} \times \mathfrak{G}_{q} \longrightarrow \mathscr{V}$, defined on morphisms in the obvious way, would give the promonoidal structure in question. The term $L(\operatorname{im}(g \circ f))$ measures the failure of the sequence (11.3) to be exact. 


\section{The dimension sequence}

Following on from Section 11, we take $F \in\left[\mathfrak{G}_{q}\right.$, Vect $\left._{\mathbb{C}}\right]$ and define its dimension sequence $\operatorname{dim} F \in$ $\mathbb{Z}^{\mathbb{N}}$ by

$$
(\operatorname{dim} F) n=\operatorname{dim}\left(F\left(\mathbb{F}_{q}^{n}\right)\right) .
$$

This inspires an algebra structure on $A^{\mathbb{N}}$ for any $k$-algebra $A$. We assume we have $\lambda \in k$ as before, but also some integer $q$ (not necessarily a prime power). As in [17], we use

$$
\phi_{n}(q)=\left(q^{n}-1\right)\left(q^{n-1}-1\right) \cdots(q-1) .
$$

We define

$$
\left[\begin{array}{c}
n \\
r, s
\end{array}\right]_{q}=\frac{\phi_{n}(q)}{\phi_{r}(q) \phi_{s}(q)}, \quad\left[\begin{array}{c}
n \\
r, s, t
\end{array}\right]_{q}=\frac{\phi_{n}(q)}{\phi_{r}(q) \phi_{s}(q) \phi_{t}(q)}, \quad \ldots
$$

For $f, g \in A^{\mathbb{N}}$, put

$$
f \cdot{ }_{q}^{\lambda} g=\sum_{r+s+t=n}\left[\begin{array}{c}
n \\
r, s, t
\end{array}\right]_{q} \lambda^{t} f(r+t) g(s+t) .
$$

The calculations of Section 11 show that this is associative at least when $A=\mathbb{Z}, q$ is a prime power and $\lambda=\operatorname{dim} L(\mathbb{F})$.

More generally, I claim $A^{\mathbb{N}}$ is an associative $k$-algebra.

Proposition 12.1. $\operatorname{dim}\left(F \otimes \otimes^{L} G\right)=\operatorname{dim} F \cdot{ }_{q}^{\lambda} \operatorname{dim} G$

\section{Acknowledgements}

I am grateful to the referees for their careful work and, in particular, for pointing out the references $[1,3,20]$. The author gratefully acknowledges the support of Australian Research Council Discovery Grant DP130101969.

\section{References}

[1] Aguiar M., Ferrer Santos W., Moreira W., The Heisenberg product: from Hopf algebras and species to symmetric functions, arXiv:1504.06315.

[2] Aguiar M., Mahajan S., Monoidal functors, species and Hopf algebras, CRM Monograph Series, Vol. 29, Amer. Math. Soc., Providence, RI, 2010.

[3] Aguiar M., Moreira W., Combinatorics of the free Baxter algebra, Electron. J. Combin. 13 (2006), R17, 38 pages, math. CO/0510169.

[4] Baxter G., An analytic problem whose solution follows from a simple algebraic identity, Pacific J. Math. 10 (1960), 731-742.

[5] Cartier P., On the structure of free Baxter algebras, Adv. Math. 9 (1972), 253-265.

[6] Chikhladze D., Lack S., Street R., Hopf monoidal comonads, Theory Appl. Categ. 24 (2010), 554-563, arXiv:1002.1122.

[7] Day B., Construction of biclosed categories, Ph.D. Thesis, University of New South Wales, 1970, available at http://www.math.mq.edu.au/ street/DayPhD.pdf.

[8] Day B., On closed categories of functors, in Reports of the Midwest Category Seminar, IV, Lecture Notes in Math., Vol. 137, Springer, Berlin, 1970, 1-38.

[9] Day B., Street R., Monoidal bicategories and Hopf algebroids, Adv. Math. 129 (1997), 99-157. 
[10] Ebrahimi-Fard K., Guo L., Free Rota-Baxter algebras and rooted trees, J. Algebra Appl. 7 (2008), 167-194, math.RA/0510266.

[11] Garner R., Street R., Coalgebras governing both weighted Hurwitz products and their pointwise transforms, arXiv:1510.05323.

[12] Guo L., Keigher W., On differential Rota-Baxter algebras, J. Pure Appl. Algebra 212 (2008), 522-540, math.RA/0703780.

[13] Joyal A., Une théorie combinatoire des séries formelles, Adv. Math. 42 (1981), 1-82.

[14] Joyal A., Foncteurs analytiques et espèces de structures, in Combinatoire énumérative (Montreal, Que., 1985/Quebec, Que., 1985), Lecture Notes in Math., Vol. 1234, Springer, Berlin, 1986, 126-159.

[15] Joyal A., Street R., Tortile Yang-Baxter operators in tensor categories, J. Pure Appl. Algebra 71 (1991), $43-51$.

[16] Joyal A., Street R., Braided tensor categories, Adv. Math. 102 (1993), 20-78.

[17] Joyal A., Street R., The category of representations of the general linear groups over a finite field, J. Algebra 176 (1995), 908-946.

[18] Kapranov M.M., Analogies between the Langlands correspondence and topological quantum field theory, in Functional Analysis on the Eve of the 21st Century, Vol. 1 (New Brunswick, NJ, 1993), Progr. Math., Vol. 131, Birkhäuser Boston, Boston, MA, 1995, 119-151.

[19] Kelly G.M., Street R., Review of the elements of 2-categories, in Category Seminar (Proc. Sem., Sydney, 1972/1973), Lecture Notes in Math., Vol. 420, Springer, Berlin, 1974, 75-103.

[20] Moreira W., Products of representations of the symmetric group and non-commutative versions, Ph.D. Thesis, Texas A\&M University, 2008.

[21] Rota G.-C., Baxter algebras and combinatorial identities. I, Bull. Amer. Math. Soc. 75 (1969), 325-329.

[22] Street R., Monoidal categories in, and linking, geometry and algebra, Bull. Belg. Math. Soc. Simon Stevin 19 (2012), 769-821, arXiv:1201.2991.

[23] Zhang S., Guo L., Keigher W., Monads and distributive laws for Rota-Baxter and differential algebras, Adv. in Appl. Math. 72 (2016), 139-165, arXiv:1412.8058. 